\title{
El papel del profesor de práctica clínica
}

\author{
F. Pérez-Peña
}

\section{Introducción}

Sir William Osler (1849-1919), médico canadiense, profesor en Montreal, Pennsylvania, Baltimore y Oxford, y autor de The Principles and Practice of Medicine, debe constituir para los internistas un punto de referencia; él fue quien afirmó que 'estudiar los fenómenos de la enfermedad sin libros es cruzar el mar sin cartas de navegación, mientras que estudiar libros sin pacientes, no es ni tan siquiera embarcar'. En otras palabras, la enseñanza teórica resulta necesaria como cuestión previa a todo conocimiento científico, pero en el caso de los estudios de medicina resultará insuficiente si no va acompañada de la enseñanza práctica, de ahí la importancia y el protagonismo del profesor de práctica clínica, función que, desempeñada durante más de cuatro décadas, nos ha proporcionado una gran satisfacción.

Sin embargo, el profesorado de prácticas se encuentra desde hace varias décadas desilusionado, carente de incentivos, de reconocimiento académico y social, e injustamente remunerado con arreglo a su alta cualificación y responsabilidad asumida, y no es equivalente a su actual situación administrativa si queremos una enseñanza eficaz. Y esto sucede, a mi juicio, porque la enseñanza de la medicina -dirigida más por políticos que por profesionales- atraviesa una grave crisis, y aunque envía a la sociedad algún que otro médico, sobre todo produce muchos 'trabajadores de la salud'.

Para que la formación práctica -y también teórica- de un médico resulte eficaz sea cual fuera el plan de estudios, el profesor de práctica clínica debe desarrollar su función en un ámbito adecuado, que a nuestro juicio exige varias premisas.

\section{Primera premisa}

Tener presente que la enseñanza de la medicina es desde antaño 'una facultad universitaria' y, por tanto, debe realizarse en una universidad que contemple que junto a las funciones docentes e investigadoras comunes a cualquier licenciatura, en el caso de la medicina debe tenerse en cuenta la función asistencial, ya que sólo se podrá enseñar la medicina que se conozca y se practique. Esta función asistencial ha sido tradicionalmente olvidada por los legisladores, y da la impresión de que el Ministerio de Educación siempre quiso 'quitarse de encima' a los hospitales clínicos.

También, que en la enseñanza de la medicina, como facultad universitaria, no debe olvidarse la función y los fines de la universidad, que no sólo es un lugar donde se elabora y transmite el saber intelectual y donde además se adquiere una profesión, sino también donde el hombre recibe la formación del 'hombre total' en el sentido de Mazzei, personas con libertad de pensamiento, sin represiones de índole social, política, económica, raciales, religiosas o de sociedades secretas; hombres que reflexionen sobre los problemas contemporáneos o sobre culturas anteriores y que, aunque de una manera parcial, sean capaces de aportar nuevas ideas que nos den una visión del pensamiento humano a través de la historia.
Profesor titular de Patología y Clínica Médica (jubilado). Facultad de Medicina. Universidad Complutense. Madrid, España.

E-mail fppena02@vodafone.es 
Respecto a la medicina, lo primero sería la formación del hombre y después, aunque a la par, la formación del médico.

La universidad debe proporcionar al estudiante la formación necesaria para seguir incorporándole, nuevos aprendizajes incluso una vez que haya abandonado las aulas.

La universidad debe incentivar la aspiración hacia el saber último de las cosas. Sin este estímulo, la ciencia queda reducida a mera técnica y no debe olvidarse que un buen médico es algo diferente a un buen técnico. La Facultad de Medicina está corriendo el riesgo de transformarse en una escuela profesional o peor aún, en una mera expendeduría de títulos, y desoír las directrices de la Comunidad Europea que señalan que los estudios de medicina se realizarán en la universidad o bajo control de la misma'. Y esto sucede, en gran parte, por la dependencia de la función docente y de los objetivos asistenciales establecidos por las autoridades sanitarias 'dueñas de los hospitales'. Tememos lo peor si se crean nuevas universidades sin infraestructura docente adecuada y donde se impartan los estudios de medicina en hospitales de dudosa acreditación universitaria. Alguien señaló (creo que fue Joaquín Costa) que 'existen demasiadas universidades para tan pocos universitarios'.

La universidad no debe ser condicionada, dirigida ni instrumentalizada por el Estado. Los profesores no deben quedar a la merced de otras fuerzas que no sean las de la sabiduría.

\section{Segunda premisa}

Con la existencia de una universidad adecuada, se necesita en nuestro criterio, un hospital con infraestructura asistencial, docente e investigadora debidamente acreditada, pues si cualquier hospital y cualquier médico sirviera para enseñar medicina se pondría en duda la existencia del profesorado numerario de las facultades de medicina y la identidad universitaria de los estudios de medicina, asumiendo que la formación del médico puede realizarse en una simple escuela profesional. Equivaldría a que un estudiante de arquitectura pudiera formarse acompañando a un arquitecto en una gran empresa constructora de viviendas, donde no cabe duda que el maestro de obra y el oficial de albañilería saben colocar magistralmente los ladrillos.
Resulta bien conocido cómo en hospitales llamados universitarios se ha pretendido enseñar medicina, a pesar de no existir más de dos o tres profesores numerarios y sin ser doctores la mayoría de los profesores asociados encargados de la enseñanza práctica.

Somos partidarios de establecer distintas categorías entre los hospitales diversos, de tal forma que los hospitales acreditados como universitarios constituyan el máximo objetivo que se debe alcanzar en la carrera docente y asistencial. Consideramos que un hospital universitario debe contemplar un organigrama peculiar y ser considerado con un rango superior al resto de hospitales. No debería valorarse de igual forma ser, por ejemplo, jefe de sección de un hospital universitario que serlo de un hospital comarcal.

Para la enseñanza de la clínica médica, el profesor debe disponer de amplias zonas de enfermería general; debiera extenderse el pensamiento de mantener en salas generales a los enfermos que no precisen atenciones muy tecnificadas o constantes. De esta forma, quedaría asegurado el campo del internista, muy apropiado para la enseñanza de la medicina general a pregraduados. Los grandes hospitales que desarrollan al máximo las especialidades en detrimento de áreas de hospitalización general no resultan, en nuestro criterio, adecuados para una asistencia eficaz (y más económica) si se tiene en cuenta el sentido unitario del enfermar. Utilizar para la enseñanza del pregrado hospitales excesivamente especializados, puede presentar al alumno (futuro médico) una visión deformada de la enfermedad y del ejercicio profesional. Resulta frecuente observar cómo en los servicios de urgencias ante un paciente con angor, crisis convulsivas o dolores óseos - por citar algunos casos vividos- se solicita la consulta del cardiólogo, neurólogo o reumatólogo, cuando la conducta ante las referidas situaciones debe ser competencia del médico generalista. Y si de un paciente diabético se tratara, el endocrino solicita consultas al cardiólogo, nefrólogo, oftalmólogo, etc., pero nunca al internista, al integrador crítico en el diagnóstico $y$ tratamiento de los pacientes o al 'consultor universal' según W. Osler. Probablemente resultaría más eficaz y económico que el internista fuera el protagonista en la dirección del estudio del referido paciente y quien solicitara una consulta con los distintos especialistas. 
En la actualidad, la mayoría de los ingresos hospitalarios tienen lugar en urgencias. En el hospital universitario, gran parte de los ingresos deberían programarse a través de policlínicas o de los centros de atención primaria.

La selección del personal de un hospital universitario debería realizarse con criterios peculiares respecto a los hospitales sin enseñanza pregraduada y exigir, por este orden, formación clínica, vocación docente, capacidad didáctica, sensibilidad científica y creatividad.

Si la Facultad de Medicina dispusiera de varios hospitales concertados, la enseñanza clínica en todos ellos deberá ser controlada por el Departamento de Medicina, que homologará programas de objetivos y pruebas de evaluación, para evitar así el 'transfuguismo' estudiantil de un hospital a otro en busca de dónde aprobar más fácilmente u obtener mayor calificación, como ha sucedido en los últimos años.

\section{Tercera premisa}

Selección rigurosa del profesorado, verificar su currículo y valorar de forma prioritaria sus aptitudes profesionales (la formación clínica no se puede improvisar, sino que es fruto de muchos años de experiencia) y, a continuación y por este orden, su vocación docente, capacidad didáctica $y$, en último término, su capacidad creativa, que no necesariamente se refleja por el número de sexenios de investigación, pues para qué sirve un profesor con todos los sexenios del mundo si no ha visto un paciente ni le conocen sus alumnos.

A propósito de los famosos sexenios, señalaremos que no disponer de ellos no significa que no se hayan publicado trabajos de cierto interés para la comunidad universitaria, sino que no han sido valorados positivamente por la comisión evaluadora, lo cual no siempre es justo, al no considerarse el contexto histórico en el que se realizaron y las revistas que entonces eran de alto impacto; además, depende mucho de la composición de la comisión evaluadora, pues por poner como ejemplo, mi última evaluación (trabajos de medicina interna y tres libros basados en la investigación realizada en el Archivo General de la Administración de Alcalá de Henares) fue juzgada negativamente por una comisión constituida por un llamado internista, un cirujano cardiovascular, un ginecólogo experto en fertilidad, un cirujano general y jagárrese el lector!, un veterinario.

Si hubieran exigido sexenios de investigación con los criterios actuales a Gregorio Marañón, Pedro Pons, Jiménez Díaz, etc. ninguno de ellos lograría la cátedra, pues publicaban en revistas que hoy día carecen de impacto alguno.

Ser profesor de medicina clínica supone tener algo más que un brillante currículo basado en 300 publicaciones (una cada 15 días) que apenas aportan algo original, supone poseer junto a una sólida formación clínica y un vasto conocimiento teórico, capacidad didáctica y saber inculcar en el joven estudiante la curiosidad por el conocimiento y la necesidad de transmitirlo.

En la actualidad, parte del profesorado de clínicas ha elegido como santuario clínico no la sala de enfermos, sino el despacho, la biblioteca, el laboratorio, la lectura de la última revista o el ordenador, cuando el auténtico santuario del médico está en el contacto directo con el paciente. El médico básico precisa 'más fonendo' (método clínico) que 'tubo de ensayo'; sólo cuando se domine el primero procede el segundo.

Es preciso que deje de valorarse la experiencia docente-asistencial como un mérito subordinado a la labor investigadora; el punto cinco de la Declaración de Edimburgo nos recuerda la necesidad de formar profesores que no sólo sean expertos en el contenido de la asignatura, y que deben premiarse las cualidades docentes y en la práctica clínica, tanto como las cualidades en investigación biomédica.

Dado el actual sistema de elección del profesorado, donde brillan por su ausencia las pruebas prácticas y se premia en demasía la investigación, somos partidarios de crear la escala docente de profesorado de clínicas, que quedaría constituida por: alumno interno, médico interno (figuras contempladas en legislaciones anteriores y de reconocida eficacia), profesor ayudante, profesor titular y catedrático de clínica médica. En esta escala, los méritos que se deben valorar serían por este orden: formación clínica, vocación docente, capacidad didáctica, sensibilidad científica y capacidad creativa, todo ello verificado públicamente y con pruebas prácticas.

Finalmente, y en lo que respecta a la selección del profesorado, es urgente la necesidad de alejar de nuestra universidad la práctica de endogamia, de la que sólo cabe esperar un empobrecimiento científico y cultural grave. 


\section{Cuarta premisa}

Modificación de las pruebas de selectividad actuales que permiten ingresar en la universidad alrededor del $90 \%$ de los alumnos presentados, lo cual nos parece excesivo si se trata de seleccionar.

El alumno de medicina debería seleccionarse de forma rigurosa en la propia facultad, considerando no sólo su capacidad intelectual y expediente académico, sino también sus cualidades personales como señala la Conferencia de Edimburgo; sólo así se evitarían alumnos con desequilibrios psicológicos (hemos vivido algunos casos) o carentes de vocación, pues no hay que olvidar que muchos alumnos de medicina ingresaron en la Facultad de Medicina por su alta nota obtenida en las pruebas de selectividad, y su vocación era muy distinta a la de médico.

En la actualidad, el alumno de medicina acepta, de forma sumisa, recibir defectuosamente la formación propia de una escuela profesional en lugar de reivindicar la que corresponde a una disciplina universitaria. Tal vez por ello, en una encuesta realizada a los alumnos de $6 .^{\circ}$ curso durante tres promociones, la mayoría ignoraba quién era Jiménez Díaz, Gregorio Marañón, Fernando de Castro, Pedro Laín, Juan Negrín, dónde nació Ramón y Cajal, por dónde transcurría el Danubio, en qué siglo tuvo lugar la Revolución Francesa o quién escribió La España invertebra$d a$, por poner algún ejemplo de la curiosidad científica e histórica del alumnado de medicina.

\section{Quinta premisa}

Modificación del sistema MIR, exigiendo mayores conocimientos clínicos para aprobar las pruebas y una rigurosa evaluación al final de la residencia.

Si de lo que se trata es de evaluar conocimientos básicos para aspirar a realizar una especialidad determinada, en mi opinión nunca estará justificado preguntar cuestiones propias de quien ya está especializado; acaso es razonable que el médico básico conozca: que la fibronectina no es propia de filamentos intermedios' (uno de los constituyentes del citoesqueleto celular), o tal vez conocer ¿qué raros síndromes producen las anormalidades de los peroxisomas?, preguntas que recojo de algunas convocatorias. Amén de numerosas cuestiones, cuyas respuestas consideradas válidas y correctas, podrían plantear un debate a todo médico que haya vivido mínimamente la profesión y no esté influido por la lectura de la última revista anglosajona.

Desde hace más de dos décadas se observa una disminución progresiva de asistencia a las salas hospitalarias por parte de los alumnos de asignaturas clínicas, quienes se preguntan: ¿para qué voy a aprender clínica si luego me van a preguntar ¿cuál es el peso molecular de la insulina o cualquier síndrome raro?

Tampoco les interesa en demasía acudir a las clases teóricas, sino que dedican su tiempo a preparar millares de preguntas tipo test, y subrayan en los distintos textos en boga (especialmente anglosajones) los párrafos de menor interés para su futuro ejercicio profesional, y además lo hacen en tecnicolor, en verde los nombres propios, en rojo la fórmula, en azul aquella estupidez, y sin adorno pictórico -y despreciados-, los conceptos fundamentales.

Resulta triste escuchar a los alumnos: 'lo que enseña el profesor en clase o en prácticas suele ser interesante, pero nada tiene que ver con los conocimientos que posteriormente le van a evaluar en las pruebas MIR. También lo es que algunos profesores, e incluso facultades, olviden los objetivos institucionales (formar un médico básico) y dediquen su función a preparar a los alumnos para superar las futuras pruebas MIR.

Las actuales pruebas MIR también influyen en la elección de la especialidad y dificultan la vocación deseada. Podríamos suponer con ironía cómo un médico con vocación ginecológica termina siendo otorrinolaringólogo, como si acaso las trompas de Falopio fueran idénticas a las de Eustaquio, y todo ello en virtud de haber obtenido una determinada calificación. Ya sin ironía, recordemos cómo las plazas de atención primaria (en teoría el genuino médico) no son actualmente ocupadas (salvo honrosas excepciones) por los primeros números, e incluso quedan plazas vacantes, $y$ algunos que consiguen la plaza se vuelven a presentar para elegir otra especialidad. Si esto es así, me parece triste que la sociedad se ponga en manos de especialistas que realizan funciones nunca deseadas.

Cumplidas estas cinco premisas, el profesor de práctica clínica estará en condiciones de ejercer su función con la máxima eficacia y poner en 
práctica su metodología docente, teniendo en cuenta que la enseñanza práctica de la medicina no tiene horarios ni vacaciones, pues tiene lugar cuando se practica el acto médico y será más completa cuanto más se viva la patología en la cabecera de los enfermos. Sólo afirmarán lo contrario quienes nunca hayan pisado una sala clínica o, si alguna vez lo han hecho, ha sido de forma protocolaria y con horario preestablecido.

El aprendizaje clínico debe ser vivencial, es decir, deberá adquirirse viviendo directamente la evolución del enfermo; es un conocimiento que nunca se olvidará. La lección leída en el texto puede olvidarse con facilidad; sin embargo, aquel caso vivido difícilmente podrá olvidarse. Es diferente explicar una enfermedad que se basa únicamente en los conocimientos adquiridos en los libros de texto, que realizar la misma enseñanza cuando se tiene experiencia de tal enfermedad, vivida en la clínica. En nuestro medio, con frecuencia se ha llegado al profesorado de medicina con conocimientos teóricos rápidamente incorporados - no vividos- pero sin el bagaje clínico necesario para impartir la enseñanza de la disciplina a la que nos referimos. No podemos comprender cómo se puede llegar a ser profesor de clínica médica a edades (varios ilustres profesores de Patología y Clínica Médica lograron la cátedra alrededor de los 25 años) en las que resulta claramente incomprensible haber vivido ni siquiera la mayor parte de los casos habituales.

Aunque tal vez resulte impopular en los tiempos que corren, somos partidarios de que la enseñanza de la clínica médica corresponda al internista con la colaboración de los especialistas. Estos últimos deben tener su protagonismo en la enseñanza del posgrado.

Somos partidarios del sistema de tutorías, grupos pequeños de estudiantes y un tutordocente seleccionado con los criterios anteriormente señalados. Dicho tutor adquiriría la responsabilidad de la formación clínica del referido grupo durante la mayor parte del período clínico, controlándose su actividad por las normas del departamento. Además, este sistema serviría para estimular a los distintos grupos docentes en alcanzar resultados mejores en las evaluaciones generales que tuvieran lugar.

En el método educativo destacamos:

- Exposición completa del acto médico. El profesor realiza con el enfermo y ante los alumnos todos los momentos del acto médico (anamnesis, exploración física, petición de datos, etc.) con comentarios y observaciones oportunas. Expondrá después la interpretación de los datos, la elaboración del diagnóstico, pronóstico y tratamiento de la enfermedad, es decir, el juicio clínico. Esto se puede realizar pasando visita en policlínicas, seminarios, etc., y ser siempre discreto en el diálogo para no alarmar al paciente con palabras improcedentes. El profesor de práctica clínica también debe enseñar a utilizar el lenguaje médico con corrección y discreción.

- Realización completa del acto médico por un alumno, dirigida por el profesor. El alumno puede estar incluido en un grupo de discusión formado por otros alumnos. Se enseña así a que el alumno discurra por su cuenta en lugar de contentarse en ver cómo discurre el profesor. Esta modalidad de clase clínica que practicamos desde hace 44 años parece sumamente útil y de las más eficaces si el profesor la realiza con vocación y pericia.

- Sesiones clínicas. Las consideramos imprescindibles en la enseñanza del pre y posgrado, pues siempre ofrecerán información respecto a metódica de pensamiento y discusión. Existen distintas modalidades de sesiones clínicas: con la exposición de casos prácticos, casos de diagnóstico 'cerrado' (desconocido para el auditorio), casos con diagnóstico previamente conocido, sesiones anatomoclínicas, casos vividos en la guardia del día anterior, etc.

- La utilidad de estas sesiones clínicas, desde el punto de vista docente, depende en gran parte de la experiencia y la capacidad expositiva del ponente, de sus cualidades didácticas y de su capacidad de síntesis y saber discernir lo útil de lo accesorio. Resulta imprescindible dedicar al final de la misma cierto tiempo para la discusión del caso, y así permite realizar preguntas o solicitar aclaraciones de algunos conceptos que hayan podido quedar poco claros.

Desgraciadamente hemos observado que en las últimas décadas hay una progresiva disminución de estudiantes y posgraduados en las distintas sesiones clínicas. A la última que asistí (Sesión mensual de Medicina Interna del Hospital), acudieron no más de 50 médicos, y en el hospital habían unos 200 estudiantes, 300 residentes y más de un centenar de 'internistas'. La razón por la que esto sucede 
tal vez se deba a no contemplarse las premisas antes expuestas, pero también pienso que no se ha realizado con eficacia el relevo del método clínico, y que las nuevas promociones de médicos desconocen la metodología de las sesiones clínicas sin saber discernir entre lo qué es una sesión bibliográfica, un seminario clínico (donde el ponente ha preparado el tema) y una sesión clínica genuina. Pensamos también que la antorcha de los médicos formados en el método clínico no se ha transmitido como debiera a las nuevas promociones que, sumergidas en la innovación tecnológica, avanzan hacia una medicina despersonalizada y poco atrayente, donde el médico tradicional acabará siendo un simple 'trabajador de la salud". Venimos denunciándolo desde hace varias décadas, pero sin ostentar poder (aunque se tenga autoridad), las ideas son palabras perdidas o caen en saco roto. Cuando se dispone de poder, aunque no se tenga autoridad, las ideas se imponen.

- Incorporación a las policlínicas de medicina interna tanto en el hospital como en los centros de atención primaria. El estudiante se familiarizará no sólo con la patología más habitual, sino también con el comportamiento humano que requiere el acto médico.

- Incorporación obligatoria a los servicios de guardia, tanto en las salas de hospitalización como en urgencias, familiarizándose con la metódica que se debe seguir desde el punto de vista diagnóstico y con la conducta terapéutica a seguir en las emergencias médicas. Este tipo de enseñanza será más eficaz cuanto mayor sea la adaptación del profesorado universitario al régimen hospitalario, de ahí la necesidad de organigramas peculiares en los hospitales universitarios, donde todos los médicos deben tener actividad docente a sus distintos niveles y oficialmente reconocida.

- Establecer el examen práctico en las asignaturas clínicas como obligatorio.

- Aprovechamiento de los programas de intercambio al máximo entre las distintas universidades nacionales y de la Comunidad Europea (Erasmus, Cajal, etc.), debidamente controlados por el departamento de medicina interna.

Si se tuvieran en cuenta los puntos anteriormente expuestos, la Universidad y la Facultad de Medicina estarían en condiciones de enviar a la sociedad un médico modélico que, por su formación, garantizaría el respeto y la consideración social. Para ello, repitámoslo una vez más, es preciso que la comunidad universitaria olvide intereses personales, soporte con dignidad presiones políticas, económicas, de grupos confesionales o sociedades secretas y se entregue por entero al bien de la institución, pues de seguir como hasta ahora, sólo lograremos engañarnos a nosotros mismos, a nuestros hijos y, lo que resulta más grave, a la sociedad. Pero todo esto ya lo señalé hace 20 años.

\section{Bibliografía}

1. Mazzei E. ¿Qué es la medicina? Buenos Aires: Columba; 1968.

2. Pérez-Peña F. Palabras perdidas (discurso para algunos médicos). Aranjuez: Doce Calles; 2002.

3. Pérez-Peña F. Los últimos clínicos de San Carlos. Madrid: Vision Net; 2005. 